\title{
Pulsar science at the Sardinia Radio Telescope
}

\author{
D. Perrodin ${ }^{1 *}$, M. Burgay ${ }^{1}$, A. Corongiu ${ }^{1}$, M. Pilia ${ }^{1}$, A. Possenti ${ }^{1}$, \\ M. N. Iacolina ${ }^{1,2}$, E. Egron ${ }^{1}$, A. Ridolfi ${ }^{3}$, C. Tiburzi ${ }^{3,4}$,

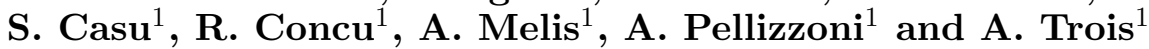 \\ ${ }^{1}$ INAF - Osservatorio Astronomico di Cagliari, 09047 Selargius (CA), Italy \\ ${ }^{2}$ Italian Space Agency, via del Politecnico snc, 00133 Roma, Italy \\ ${ }^{3}$ Max-Planck-Institut für Radioastronomie, Auf dem Huegel 69, D-53121 Bonn, Germany \\ ${ }^{4}$ Fakultät für Physik, Universität Bielefeld, Postfach 100131, D-33501 Bielefeld, Germany \\ *email: delphine@oa-cagliari.inaf.it
}

\begin{abstract}
The Sardinia Radio Telescope (SRT) is a modern, fully-steerable 64-m dish located in San Basilio, Sardinia (Italy). It is characterized by an active surface that allows it to cover a wide range of radio frequencies (300 MHz to $100 \mathrm{GHz}$ ). During SRT's commissioning phase, we installed the hardware and software needed for pulsar observations. Since then, SRT has taken part in Large European Array for Pulsars and European Pulsar Timing Array observations for the purpose of gravitational wave detection. We have installed a new S-band receiver that will allow us to search for pulsars in the Galactic Center. We also plan to combine our efforts to search for Extraterrestrial Intelligence (SETI) with the search for pulsars and Fast Radio Bursts.
\end{abstract}

Keywords. pulsars:general, gravitational waves

\section{Introduction}

SRT was officially inaugurated in 2013, and the technical and scientific commissioning work was completed in late 2015. An early-science run was offered to the community in the first half of 2016. Following the upgrade of its active surface, SRT is currently undergoing a second phase of technical and scientific tests, and will again be offered to the scientific community in late 2018. In the near future, pulsar observations will be available in P-band (305-410 MHz), L-band (1.3-1.8 GHz), S-band (3-4.5 GHz), C-band (5.7-7.7 GHz), and K-band (18-26 GHz). The available pulsar backends include a Parkes Digital Filberbank (PDFB3), two ROACH1 backends, as well as the SArdinia Roach2 Digital Architecture for Radio Astronomy (SARDARA) system, which consists of seven ROACH2 boards running on Graphics Processing Units (GPUs).

\section{Pulsar timing at SRT}

We have so far tested the telescope's pulsar timing capabilities at P-band, L-band and C-band with the PDFB3 and ROACH1 backends (Prandoni et al. 2017). The development of the SARDARA system for pulsar observing is currently ongoing. SRT is part of the European Pulsar Timing Array (EPTA) collaboration and in particular of the Large European Array for Pulsars (LEAP) project. Pulsar timing arrays seek to detect nanohertz-frequency gravitational waves, such as those from inspiralling supermassive black hole binaries or from cosmological origins, by monitoring the radio emission from an array of pulsars over many years and searching for deviations in the expected arrival times of the radio pulses (Lentati et al. 2015). The LEAP project involves the monthly, simultaneous observing of $\sim 20$ millisecond pulsars (MSPs) at five large European radio 
telescopes, followed by the coherent addition of the pulsar data (Bassa et al. 2016, Smits et al. 2017). Since the beginning of the scientific commissioning phase, and during the early-science run (2016), SRT has taken part in the LEAP project using the L-band receiver and the ROACH1 backend. SRT is the the latest addition to the LEAP project; the achieved sensitivity with all five telescopes is equivalent to that of a 196-meter dish (Perrodin et al. 2016, Prandoni et al. 2014). During the same time period, we have performed L-band and P-band observations for EPTA timing purposes, with both the DFB and the ROACH1 backends. SRT's LEAP and EPTA observations will greatly enhance the efforts to impose stringent limits on a gravitational wave background from supermassive black hole binaries. Early-science projects (2016) have also involved the monitoring of double neutron star systems for the purpose of testing strong gravity, as well as eclipsing binaries, using the dual L-P band receiver. This receiver allows us to simultaneously observe in $\mathrm{L}$ and $\mathrm{P}$ bands, making it the ideal instrument with which to observe eclipsing binaries, and in particular to study the mechanism that generates eclipses.

\section{A new multi-feed S-band receiver (3-4.5 GHz)}

A multi-feed S-band receiver has been funded by the Sardinia region (PI: Iacolina, 2013). The receiver with one central feed was completed and tested in late 2016 (Valente et al. 2014, Valente et al. 2016), and bright known pulsars were successfully detected. In the next few years, the receiver will have seven feeds and will be the only single-dish, multi-beam S-band receiver in the world. The seven beams will be connected to seven ROACH2 boards (the SARDARA system) and GPUs. This receiver will be used for EPTA timing at S-band, along with the Effelsberg and Nançay telescopes, allowing for a good determination of dispersion measure corrections in the SRT and EPTA datasets. Additionally, this receiver will be used to perform pulsar searches in the Galactic Center. The S-band is ideal for Galactic Center surveys since the higher frequencies allow the observing system to overcome dispersion effects that are problematic at lower frequencies, yet the expected flux is still elevated at these frequencies. With a small sampling time (50 microseconds) and a high number of frequency channels (4096), we could detect MSPs in tight orbits in the Galactic Center. The discovery of MSPs close to the Galactic Center would be especially interesting for tests of gravity, since the timing of these pulsars would allow us to probe the environment around the central supermassive black hole ( $\left.\operatorname{Sgr} \mathrm{A}^{*}\right)$.

\section{SETI, pulsar searching and Fast Radio Bursts}

Through a collaboration with the Breakthrough Listen program, we plan on implementing the SERENDIP VI setup, using the SARDARA backend and GPUs. This setup will allow us to collect data, either with targeted observations or in piggy-back mode, and search simultaneously for extraterrestrial intelligence, pulsars and Fast Radio Bursts.

\section{References}

Bassa, C. et al. 2016, MNRAS, 456, 2196, arXiv: 1511.06597

Lentati, L. et al. 2015, MNRAS, 453, 2576, arXiv: 1504.03692

Perrodin, D. et al. 2016, Proc. of MG14, arXiv:1608.01839

Prandoni, I. et al. 2017, A\& A, accepted, arXiv:1703.09673

Prandoni, I et al. 2014, Proc. of 12th European VLBI Network Symposium and Users Meeting

Smits, R. et al. 2017, Astronomy and Computing, 19, 66, arXiv:1703.06438

Valente, G. et al. 2016, Proceedings of the SPIE, 9914, 991422

Valente, G. et al. 2014, Proceedings of the SPIE, 9153, 91530Q 